\title{
Reports
}

\section{Rapid quantification of inflammation in tissue samples using perfluorocarbon emulsion and fluorine-19 nuclear magnetic resonance}

\author{
Eric T. Ahrens, Won-Bin Young, Hongyan Xu, and Lisa K. Pusateri \\ Department of Biological Sciences, Carnegie Mellon University, Pittsburgh, PA, USA
}

BioTechniques 50:229-234 (April 2011) doi 10.2144/000113652

Keywords: inflammation; macrophage; EAE; perfluorocarbon; emulsion; ${ }^{19} \mathrm{~F} ; \mathrm{NMR}$

Quantification of inflammation in tissue samples can be a time-intensive bottleneck in therapeutic discovery and preclinical endeavors. We describe a versatile and rapid approach to quantitatively assay macrophage burden in intact tissue samples. Perfluorocarbon (PFC) emulsion is injected intravenously, and the emulsion droplets are effectively taken up by monocytes and macrophages. These 'in situ' labeled cells participate in inflammatory events in vivo resulting in PFC accumulation at inflammatory loci. Necropsied tissues or intact organs are subjected to conventional fluorine-19 $\left({ }^{19} \mathrm{~F}\right)$ NMR spectroscopy to quantify the total fluorine content per sample, proportional to the macrophage burden. We applied these methods to a rat model of experimental allergic encephalomyelitis (EAE) exhibiting extensive inflammation and demyelination in the central nervous system (CNS), particularly in the spinal cord. In a cohort of EAE rats, we used ${ }^{19} \mathrm{~F}$ NMR to derive an inflammation index (IFI) in intact CNS tissues. Immunohistochemistry was used to confirm intracellular colocalization of the PFC droplets within CNS CD68+ cells having macrophage morphology. The IFI linearly correlated to mRNA levels of CD68 via real-time PCR analysis. This ${ }^{19} \mathrm{~F}$ NMR approach can accelerate tissue analysis by at least an order of magnitude compared with histological approaches.

Rapid, quantitative scoring of inflammation in tissue specimens is a common need in many facets of biomedical research. Discovery and preclinical studies often rely on histological processing and analysis of a panel of tissues obtained from animal models, and these procedures are often viewed as timeconsuming and expensive, and a bottleneck in research and development endeavors. Here, we describe a platform to rapidly and quantitatively assay macrophage infiltration in intact tissue samples. In this approach, we utilize a perfluorocarbon (PFC) emulsion reagent that labels phagocytic monocytes and macrophages in vivo, and conventional fluorine-19 $\left({ }^{19} \mathrm{~F}\right)$ nuclear magnetic resonance (NMR) spectroscopy is used as a quantitative readout of inflammation in intact, excised tissue samples. The approach requires no tissue preparation other than an optional fixation step.

Assaying inflammation often involves tissue-destructive methods. Commonly, thinsectioning $(4-10 \mu \mathrm{m})$ of embedded tissues is used in combination with one of more stains, for example, using colorimetric hematoxylin and eosin (H\&E), or using immunoreactive reagents that highlight cell-specific markers or molecular events. Organ analysis of inflammation often requires serial sectioning and the preparation of a large number of slides. Periodic sampling of serial sections for the sake of accelerating analysis may lead to bias or quantification inaccuracies, or may necessitate a large experimental group to achieve statistical significance. Thus, widespread biodistribution analysis of a large panel of tissues taken from a single animal-much less an experimental group - can be a laborious undertaking. Although great strides have been made to increase tissue histology throughput, section preparation and analysis can only be made semiautomated at this time.

Alternatively, 'bulk' cellular, biochemical, or molecular analyses of disrupted tissue samples have been developed to assay inflammation. For example, approaches include monitoring the expression levels of selected biomarkers in peripheral blood mononuclear cells using flow cytometry (1), mRNA profiling using real-time PCR, or ELISA to detect monocyte-specific markers or pro-inflammatory cytokines $(2,3)$.

In this article, we describe an alternative approach to assay inflammation (Figure 1), without the need for tissue disruption, thereby potentially accelerating studies. The approach uses a PFC emulsion reagent comprised of a colloidal suspension of non-toxic PFC formulated into small ( 150-nm diameter) emulsion droplets. Following i.v. injection, the droplets are taken up by phagocytic cells (4-7), predominately monocytes and macrophages, and to a small degree neutrophils. These 'in situ' labeled cells subsequently participate in inflammatory events in vivo, resulting in an accumulation of PFC at inflammatory loci. Necropsied tissues samples, or intact organs, are subjected to conventional ${ }^{19} \mathrm{~F}$ NMR analysis to quantitatively measure the total fluorine content per sample, which is proportional to the inflammatory burden. There is negligible natural abundance of ${ }^{19} \mathrm{~F}$ in soft tissues, thus this approach offers high specificity to inflammation.

PFC-based reagents are biologically safe, even at very high doses. PFC emulsions have been studied clinically for many years as potential artificial blood substitutes in humans $(8,9)$, although the PFC molecule used in the present study is different than those used for blood substitutes and has been optimized for ${ }^{19} \mathrm{~F}$ NMR-MRI applications. Numerous in vitro studies have shown that 


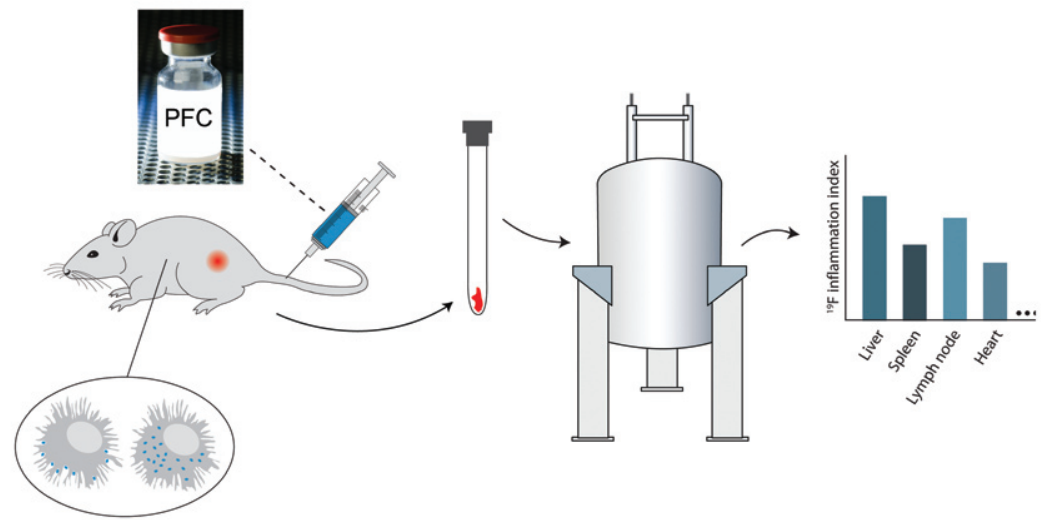

Figure 1. Overview of inflammation quantification of intact tissue samples using ${ }^{19} \mathrm{~F} \mathrm{NMR}$. PFC emulsion is injected i.v. and is taken up by monocytes and macrophages. These labeled cells participate in inflammatory events in vivo resulting in an accumulation of ${ }^{19} \mathrm{~F}$ at inflammatory loci. Conventional ${ }^{19} \mathrm{~F}$ NMR spectroscopy of panels of intact tissue samples is used to measure histogram profiles of the inflammatory index (IFI), proportional to tissue macrophage burden.

intracellular labeling with PFC does not affect cell phenotype and function (10-14).

As an example of the applicability of these methods, we deliver PFC emulsion reagent intravenously to the dark agouti (DA) rat model of experimental allergic encephalomyelitis (EAE) (15), a widely used model for human multiple sclerosis (MS). EAE has many clinical and histopathological similarities to MS (16). EAE is most commonly induced by immunizing animals with myelin proteins or their disease-inducing peptides (16). Clinical disease develops when primed $\mathrm{CD}^{+} \mathrm{T}$ cells enter the central nervous system (CNS) and recognize their cognate self-antigen presented in the context of MHC class II molecules. The resulting perivascular and parenchymal infiltrations in the CNS, particularly in the spinal cord, include a large number of macrophages and eventually lead to demyelination and clinical paralysis. In the EAE model, the ability to rapidly assay the inflammatory biodistribution in the CNS would be tremendously beneficial in attempts to understand the pathogenesis of autoimmunity and to design therapeutic interventions. Overall, the methods described herein can dramatically reduce the time to evaluate macrophage involvement in a wide variety of acute and chronic inflammatory models.

\section{Materials and methods}

\section{EAE model and PFC administration}

Experiments were carried out in accordance with the guidelines provided by the Carnegie Mellon Institutional Animal Care and Use Committee (IACUC) and the National Institute of Health Guide for the Care and Use of Laboratory Animals. The EAE rat model was generated using previously reported methods (15). Adult female DA rats (Harlan, Indianapolis, IN, USA), 11 weeks old, were inoculated with emulsified syngeneic spinal cord. The inoculant was prepared by homogenizing frozen DA spinal cord ( $50 \mathrm{mg} / \mathrm{rat}$ ) with incomplete Freud's adjuvant (IFA; cat. no. DF0639-60-6; Difco, Detroit, MI, USA) at $200 \mu \mathrm{L} / \mathrm{rat}$ and Mycobacterium tuberculosis (H37Ra; cat. no. DF3114-33-8; Difco) at $2 \mathrm{mg} / \mathrm{mL}$. The rat's tail base was shaved, and a single injection of the homogenate was delivered subcutaneously in a total of $n=11$ rats. A control group of rats $(n=3)$ were also prepared by inoculating with adjuvant in the same manner, except spinal cord homogenate and H37Ra were excluded. After induction, rats were monitored daily for body weight and clinical symptoms of EAE. Symptoms were scored according to the convention: $0=$ normal; $1=$ limp tail; 2 = paraparesis with a clumsy gait; $3=$ hindlimb paralysis; $4=$ hind- and forelimb paralysis; $5=$ moribund.

Upon the first signs that an animal reached clinical stage 2-typically 16-20 days after induction in $93 \%$ of the subjects-rats received a single i.v. injection $(0.5 \mathrm{~mL})$ of PFC emulsion (Cat. no. VS-580; Celsense, Inc., Pittsburgh, PA, USA) via the jugular vein. This sterile reagent contains emulsified perfluoro-15crown- 5 ether at a volume fraction of $20 \%(\mathrm{v} / \mathrm{v})$, and a mean droplet size of $145 \mathrm{~nm}$. Two days after injection with PFC rats were sacrificed.

In a subset of animals $(n=4)$ the PFC emulsion was rendered fluorescent prior to injection to aid in histological analysis. This was achieved via adding a simple premix step of the PFC emulsion with a lipophilic dialkylcarbocyanine (DiI; cat. no. V22885; Molecular Probes, Inc., Eugene, OR, USA) fluorophore. The fluorescent inoculant was prepared by mixing $5 \mathrm{~mL}$ emulsion with a $2-\mu \mathrm{L} / \mathrm{mL}$ DiI stock solution prepared according to the manufacturer's instructions. After incubating the emulsion with Dil for $20 \mathrm{~min}$ at room temperature under gentle agitation, the $\mathrm{DiI}$ molecules became associated with the PFC emulsion droplets
(14). The fluorescent emulsion was then injected into the stage $2 \mathrm{EAE}$ rat.

Tissue preparation for NMR

Two days after PFC injection, rats $(n=8)$ were anesthetized and sacrificed by transcardial perfusion with PBS followed by $4 \%$ paraformaldehyde (PFA). The intact dorsal columns and brain were excised and stored in $4 \%$ PFA for $>24 \mathrm{~h}$. Using a razor blade, the entire dorsal column, except the sacral region, was partitioned at intervertebral discs into a total of 15 segments, where each segment contained 1-3 vertebrae. The spinal cord was dissected from each segment, discarding the vertebral bone and spinal nerves, and each segment was weighed. Using a scalpel, the intact brain stem and cerebellum was also dissected from the excised brain and weighed. Other organs were also necropsied and fixed in control rats for PFC biodistribution analysis.

\section{${ }^{19}$ F NMR}

Fixed CNS samples and other tissues from EAE and control rats were subjected to ${ }^{19} \mathrm{~F}$ NMR analysis. The weighed tissue samples were placed at the bottom of quartz 5-mm NMR tubes (Cat. no. WG-1000; WilmanLabglass, Vineland, NJ, USA). Importantly, the specimen size was small enough to fit entirely within the homogenous receptive field of the NMR probe so that all ${ }^{19} \mathrm{~F}$ was detected. (The spinal cord segments of $1-3$ vertebrae in length, brain stem, and cerebellum easily met this criterion.) A $10-\mu \mathrm{L}, 2 \%(\mathrm{v} / \mathrm{v}$ ) aliquot of trifluoroacetic acid (TFA) was placed in a flame-sealed $1 \mathrm{~mm}$-diameter glass capillary tube (Cat. no. 34507-99; Kimble Kontes, Vineland, NJ, USA) and positioned inside the NMR tube next to the tissue sample; this TFA reference contained a total of $N_{F}=1.2 \times 10^{19}$, where $N_{F}$ is the number of fluorine atoms. A one-dimensional ${ }^{19} \mathrm{~F}$ NMR spectrum was acquired for each sample at $470 \mathrm{MHz}$ using a Bruker spectrometer (Bruker Biospin, Billerica, MA, USA) with a delay time of 10 $s$ and 32 averages. Two distinct peaks were observed at approximately $-76 \mathrm{ppm}$ for TFA and -92 ppm for the PFC. The total fluorine content $\left(C_{F}\right)$ of the sample was calculated from the integrated peak areas for the PFC $\left(I_{P F C}\right)$ and TFA $\left(I_{T F A}\right)$ reference using the formula $C_{F}$ $=I_{P F C} N_{F} / I_{T F A}$. Results were normalized to the tissue weight $(w)$, yielding an inflammation index $(I F I)$ of $I F I=C_{F} / w$, expressed as the number of ${ }^{19} \mathrm{~F}$ atoms per gram of tissue.

\section{Histology and immunohistochemistry} After NMR analysis, selected spinal cords were paraffin-embedded and sectioned at $10-\mu \mathrm{m}$ thickness and stained with H\&E using standard procedures. Immumohistochemistry was also performed on selected spinal cords that were flash-frozen in OCT compound 
(Qiagen, Valencia, CA, USA) and cryosectioned at $6-\mu \mathrm{m}$ thickness. Sections were stained for CD68 using ED1 as the primary antibody (MCA341R; AbD Serotec, Inc., Raleigh, NC, USA), followed by a secondary horse anti-mouse antibody conjugated to FITC dye (Cat. no. FL-2000; Vector Laboratories, Burlingame, CA, USA). We also stained for astrocytes using rabbit anti-GFAP (Cat. no. ab7260; Abcam, Inc., Cambridge, MA, USA) follow by a secondary Alexa Fluor488conjugated, donkey anti-rabbit antibody (Cat. no. A21206; Invitrogen, Carlsbad, CA, USA). Stained sections were imaged using a Zeiss 510 LSM UV Duoscan microscope (Carl Zeiss MicroImaging, Thornwood, NJ, USA).

\section{Real-time PCR}

In selected EAE rats $(n=3)$, fresh spinal cord segments were retrieved and partitioned, as above. The tissue segments were then further cut in half, where one part was fixed and subjected to ${ }^{19} \mathrm{FNMR}$ measurement as described in the "Fi9 NMR" section, and the other half was preserved in RNAlater (Ambion, Austin, TX, USA) for real-time PCR analysis. Total RNA was extracted for the PCR samples using TRIzol (Invitrogen) following the manufacturer's instructions. From each sample, $2 \mu \mathrm{g}$ total RNA were reverse-transcribed into first strand cDNA with SuperScript III (Invitrogen) and poly-T primers. Multiplexed, real-time PCR was performed on a Stratagene MX3000 (Stratagene, La Jolla, CA, USA) to evaluate the expression of CD68, a biomarker for monocytes and tissue macrophages, as well as astrocyte-specific glial fibrillary acidic protein (GFAP). Expression levels of CD68 and GFAP in each sample were normalized to expression of the 'housekeeping gene' glyceraldehyde 3-phosphate dehydrogenase (gapdh). The primer sequences are listed below (where $F$ is a forward primer and $R$ is a reverse primer): CD68F:5'-GCATAGTTCTTTCTCCAGCAATTCACCT-3', CD68 R: 5'-GAGAGGCAGCAAGAGAGATTGGTCA-3', and CD68 probe: 5'-FAM-AGGGACACTTCGGGCCATGCTTCTCTT-Iowa FQ; GFAP F: 5'-GCCTTGACCTGCGACCTTGAGT-3', GFAP R: 5'-GTGCCTCCTGGTAACTCGCCG-3', and GFAP probe: 5'-Cy5-CGGCACGAACGAGTCCTTGGAGAGGC-IAbRQSp-3'; GAPDH F: 5'TGAACGGGAAGCTCACTGGCAT-3', GAPDH R: 5'-CGCCTGCTTCACCACCTTCTTG-3', and GAPDH probe: 5'-HEX-CCGCCTGGAGAAACCTGCCAAGTATGATGAC-Iowa FQ.

\section{Results and discussion}

We used NMR measurements on the tissue samples to quantify macrophage burden.
Figure $2 \mathrm{~A}$ displays a typical ${ }^{19} \mathrm{~F}$ spectrum of a spinal cord segment; it shows a single PFC peak at $-92 \mathrm{ppm}$ and a TFA peak at $-76 \mathrm{ppm}$ from the sealed reference capillary placed alongside the sample. From such data, we calculated the IFI along the length of the spinal cord (Figure 2B), where the IFI values represent the apparent number of ${ }^{19} \mathrm{~F}$ nuclei per tissue weight. These data represent the mean of $n=8$ spinal cords, where the vertebrae are enumerated along the abscissa. The ${ }^{19} \mathrm{~F}$ content of the tissueand thus IFI-is linearly proportional to the amount of macrophage present in the tissue. The IFI results (closed circles, Figure 2B) are consistent with the known topology of the inflammation distribution in the CNS in this EAE rat model, which is particularly prevalent in the spinal cord (15). The EAE CNS, especially the spinal cord, is generally the focus of study when evaluating therapeutic candidates for MS.

The control group (open circles, Figure 2B) receiving a CFA injection, but no spinal cord homogenate, showed minimal ${ }^{19} \mathrm{~F}$ uptake in the CNS, indicating that the PFC emulsion does not cross the intact blood-brain barrier or enter into the CNS in the absence of autoimmune disease.

Overall, in these experiments the total preparation and analysis time per rat CNS was $\sim 6 \mathrm{~h}$, which is vastly accelerated compared with most conventional histological analyses. Moreover, utilization of robotic or automated NMR sample-changing devices, commonplace in many NMR laboratories, can enable rapid data acquisition of tissue panels.

The PFC emulsion droplets are generally taken up by the reticuloendothelial system (RES) (4-7). This behavior was confirmed by ${ }^{19}$ F NMR using excised tissues and organs from a control DA rat (Figure 2C).

To validate the cell type incorporating the PFC emulsion droplets, the same tissues were examined histologically following NMR. Figure $3 \mathrm{~A}$ shows $\mathrm{H} \& \mathrm{E}$ staining in the EAE (left panel) and control (right panel) cervical spinal cord white matter; the panels show the characteristic EAE pathology with a large number of perivascular mononuclear infiltrates compared with control.

Using immunohistochemistry in the EAE spinal cord, we also confirmed that the PFC emulsion droplets colocalized within CD68+ cells that have morphologies consistent with monocytes and macrophages. For these experiments, we used stage 2 EAE rats that were injected with pretreated PFC emulsion such that the droplets had surface-bound fluorescent DiI. The fluorescent microscopy images of white matter regions of the spinal cord display punctate deposits of DiI (red, Figure 3B) within immunostained CD68+ cells (green, Figure 3B) consistent with

\section{PGR Tthes \\ apalas \\ Uniformly Reliable}

BRAND PCR tubes, strips and plates are precision molded from virgin resins for excellent, reproducible results in most $\mathrm{PCR}^{*}$ applications.

- Thin, uniform walls ensure consistent thermal transfer for excellent results

- Autoclavable and RNase-, DNase-, and DNA-free

- Compatible with most thermal cyclers

- Individual tubes have attached caps

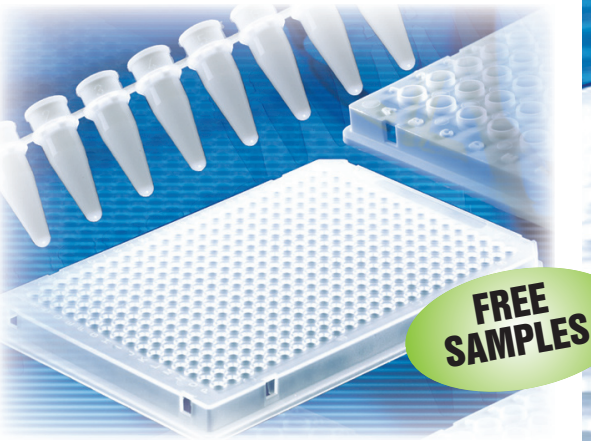

NEW! White PCR tubes, strips and plates for Real Time PCR (qPCR) offer significantly better results in the analysis of fluorescence signals.

*The Polymerase Chain Reaction (PCR) is covered by international patents. Use of the PCR process may require a license.

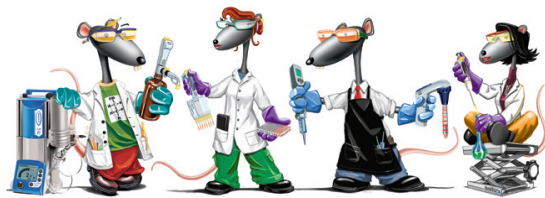

Lab Rats Trust BrandTech!

BRANDTECH SCIENTIFIC, INC.

Toll Free (888) 522-2726 www.brandtech.com 
A

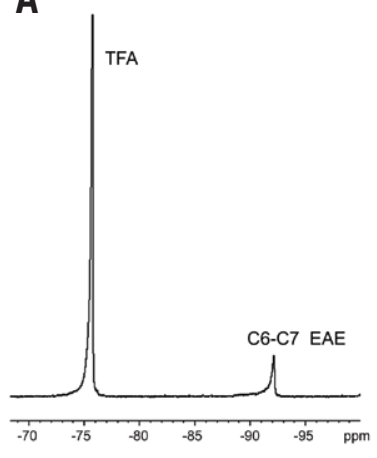

B

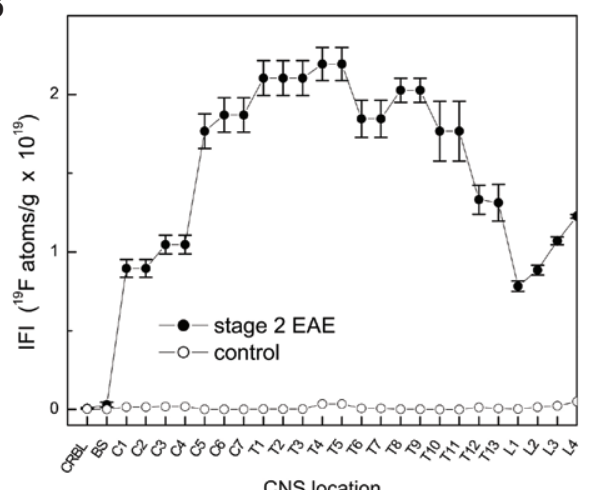

C

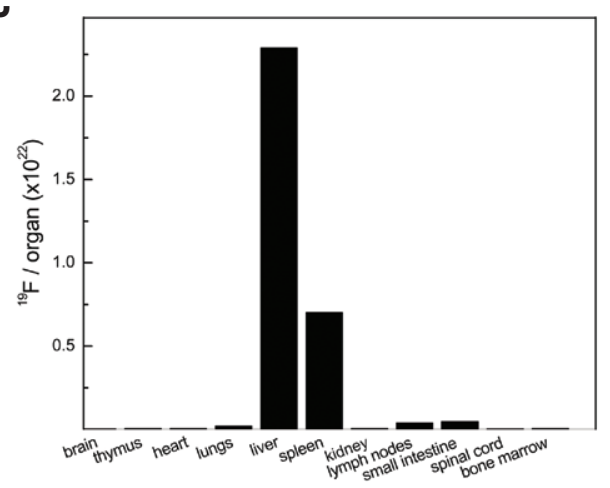

Figure 2. NMR results in the EAE (stage 2) and control rats. (A) ${ }^{19} \mathrm{~F} N M R$ spectrum of a fixed, intact cervical spinal cord segment from EAE rat. The PFC displays a single peak at -92 ppm and the TFA reference peak is at -76 ppm. (B) Average inflammation index (IFI) along spinal cord axis in EAE ( $n=8$ ) and control $(n=3)$ rats (closed and open circles, respectively). CRBL, cerebellum; BS, brainstem. The IFI is expressed as the number of ${ }^{19} \mathrm{~F}$ atoms per gram of tissue. (C) ${ }^{19} \mathrm{~F}$ NMR measurements of organ and tissue biodistribution of the PFC emulsion in a control rat shows RES uptake.

A
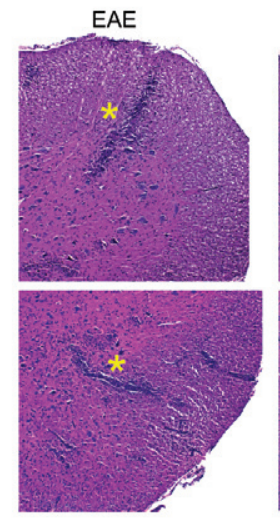

control

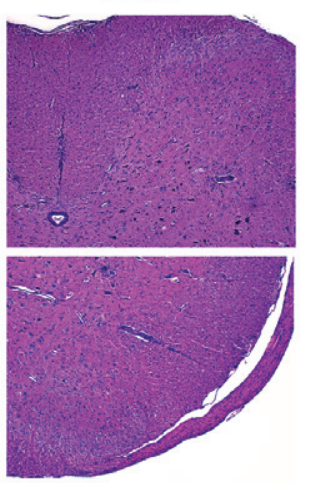

B

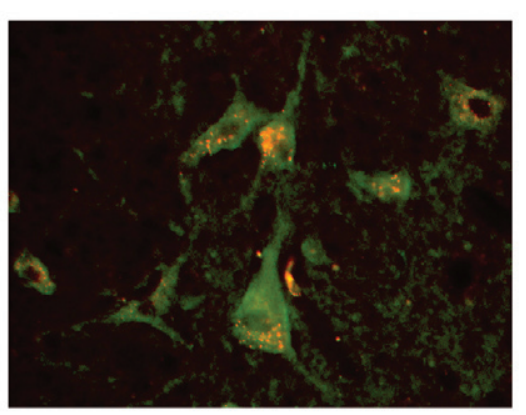

C

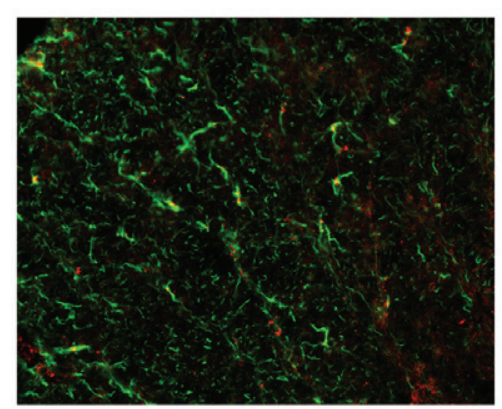

Figure 3. Histological sections of cervical EAE (stage 2) and control spinal cords. (A) H\&E stained section (10x) in EAE (left panel) and control (right panel) spinal cords, where the top and bottom panels are dorsal and ventral white matter, respectively. EAE pathology (asterisks) shows significant perivascular infiltrates (blue) compared with control. (B) Fluorescent images (40x) of immunostained white matter regions showing punctate deposits of PFC-Dil (red) within CD68+ cells (green). Data show that PFC droplets colocalized within CD68+ cells that have morphologies consistent with monocytes and macrophages. (C) Fluorescent micrograph (40x) showing an absence of Dil-PFC (red) colocalization in GFAP-stained cells (green).

perinuclear vesicular localization of the PFC. The intracellular localization of PFC is reminiscent of what has been previously observed in dendritic cells $(14,17)$. In contrast, DiI-PFC was not observed in glial cells that were immunostained for GFAP (Figure 3C). This indicates that no apparent PFC was taken up by astrocytes.

To confirm that the IFI linearly correlates to the density of monocyte/macrophage, we conducted quantitative PCR studies in EAE CNS tissue. Figure 4A shows a representative correlation analysis from a single stage 2 EAE rat, where the ordinate displays IFI obtained from NMR, and the abscissa displays the CD68 mRNA levels normalized to the GAPDH housekeeping gene. A linear regression analysis yields an $R$ correlation coefficient of 0.89 , showing a high degree of linearity between IFI and CD 68 levels (Figure 4A). Overall, the average $R$ correlation coefficient calculated for all animals studied $(n=3)$ was 0.68 .

Since astrocytes can also express low levels of CD68, to rule out their involvement in
PFC uptake we also performed real-time PCR with the astrocyte-specific marker GFAP in a multiplexed, real-time PCR reaction along with CD68 and GAPDH. We did not observe any correlation between GFAP and ${ }^{19} \mathrm{~F}$ uptake $(R=-0.13$; Figure $4 \mathrm{~B})$.

The elevation of the CD 68 mRNA level in EAE spinal cord was $\geq 100$-fold higher compared with spinal cord samples from control rats (data not shown), where only minimal CD 68 mRNA levels were detected. Thus, CD68 expressed in astrocytes and other glial cells is relatively low, and the CD68 detected in EAE spinal cord was mainly contributed by EAE-associated monocyte or macrophage infiltrates. The mRNA profiling data, in combination with the histological observation of PFC localization only within CD68+ cells with monocyte-macrophage morphology, suggest that PFC uptake and the measured IFI reflects the inflammation burden.

The PFC molecules used are not degraded in vivo by any known enzyme or at low $\mathrm{pH}(5,6)$. The ${ }^{19} \mathrm{~F}$ isotope has $100 \%$ natural abundance and is stable. In contrast, ${ }^{18} \mathrm{~F}$-based positron emission tomography (PET) probes used in EAE studies have a limited half-life (18). The blood half-life of the PFC emulsion formulation used is $\sim 9.4$ $\pm 2.6 \mathrm{~h}$ in a DA rat (19), but may depend on species/strain and inflammation status of the subject. As shown in the control rats, the emulsion does not appear to cross the intact blood-brain barrier. The PFC emulsion is taken up by macrophages and monocytes, possibly in blood, and then carried into inflammatory loci. Generally, clearance of the PFC emulsion is via the reticuloendothelial system and exhalation through the lungs (4-6).

Prior work has reported the use of in vivo labeling and imaging of macrophage using PFC emulsion in various animal models (7,20-22), including early work in an EAE model (7); these studies mainly focus on the use of in vivo ${ }^{19} \mathrm{~F}$ MRI techniques. However, small animal MRI instruments are much less commonplace compared with ubiquitous NMR spectrometers. Moreover, the cell 
A

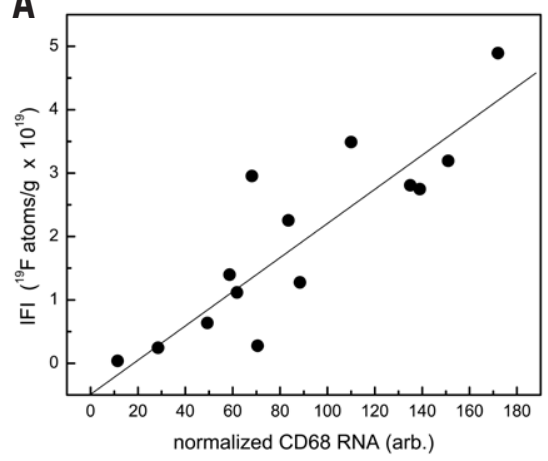

B

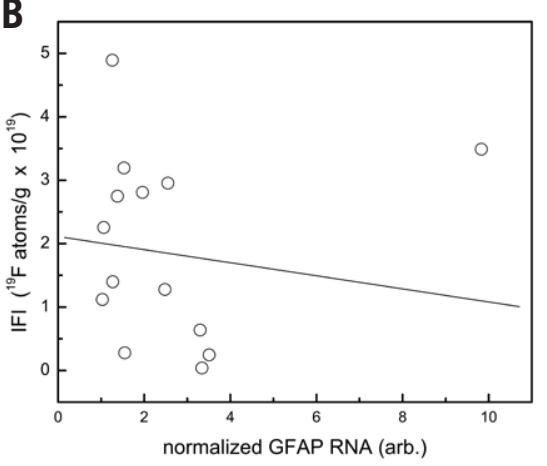

Figure 4. Quantitative analysis of macrophage burden in EAE spinal cord tissue using real-time PCR in a single rat. (A) Correlation between IFI, obtained from ${ }^{19} \mathrm{~F} \mathrm{NMR}$, and CD68 mRNA levels. Data shows a linear correlation $(R=0.89)$, consistent with the PFC internalization into macrophage. The mRNA levels are normalized to gapdh. (B) Plot of IFI versus GFAP mRNA shows no correlation (R=-0.13), suggesting that PFC is not taken up by astrocytes.

detection sensitivity of high-field NMR instruments greatly exceeds that of MRI instrumentation for detection of sparse cell numbers. We estimate that one can detect on the order of $10^{3}$ cells per sample using highfield NMR instrumentation (e.g., 11.7 T), whereas high-field MRI detection limits are closer to $10^{3}-10^{4}$ cells per voxel (13).

This study used tissue samples that were small enough to fit inside a standard 5-mm NMR tube and remain in the homogenous region of NMR coil. In mouse, intact organ tissues (spleen, lymph nodes, thymus, etc.) meet these criteria. For larger tissue samples (midbrain, heart, liver, etc.), several options are available: $(i)$ the total organ or tissue can be manually segmented into discrete samples, each measured separately [this is the approach that we used for our study of CNS tissues (Figure 2B)]; (ii) one can dissect and weigh a piece of the whole tissue or organ of interest that fits into the NMR tube and use this for the IFI measurement [this approach assumes that the inflammation (i.e., ${ }^{19} \mathrm{~F}$ ) distribution is roughly homogenous throughout the whole tissue or organ]; (iii) a tissue homogenate can be made by mechanical disruption, and a weighed aliquot is placed in the NMR tube to assay IFI of the sample; (iv) one can use larger-diameter NMR hardware (i.e., NMR tube and probe); for example, a standard 10-mm NMR tube can readily accommodate an intact mouse brain.

These same whole-tissue NMR methods can also be useful for 'counting' transplanted therapeutic or diagnostic cells that have been labeled with PFC emulsion ex vivo prior to transfer in vivo (13,23). A multitude of regenerative medicine and immunotherapeutic cell therapy studies could benefit from a tool that does not rely on laborious histology or flow cytometry to accurately assay cell biodistribution in tissue panels. Moreover, ex vivo labeled, phenotypically defined, immune cells can also be used for inflammation diagnostic purposes when infused into a subject. Using ex vivo labeling approaches, the mean PFC uptake, expressed as ${ }^{19} \mathrm{~F} / \mathrm{cell}$, is often measured as part of the cell labeling protocol development (13), and this parameter can be used to calculate the apparent number of cells present in tissue samples directly from the ${ }^{19}$ F NMR data $(13,23)$.

In summary, we show that it is feasible to rapidly assay monocyte/macrophage burden in intact tissues using PFC emulsion administered in vivo and a conventional NMR scanner. These methods can be used to accelerate routine analysis of inflammation in discovery and preclinical studies.

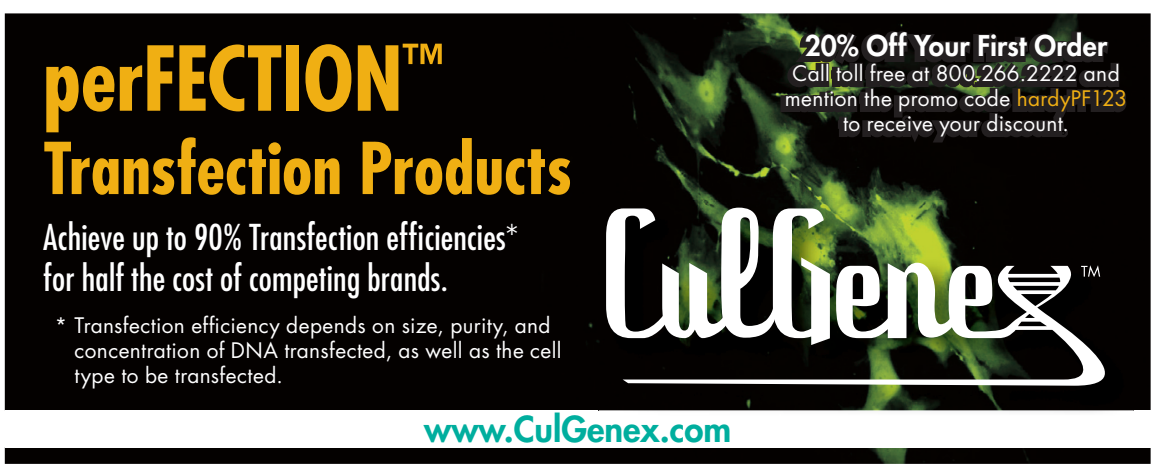

\section{Acknowledgments}

We thank Kevin Hitchens and Virgil Simplaceanu for helpful discussion. This work was supported by the National Institutes of Health (NIH; grant nos. R01-CA134633, R01-EB003453, P01-HD047675, P41EB001977). This paper is subject to the NIH Public Access Policy.

\section{Competing interests}

E.T.A. is a consultant for Celsense, Inc. All other authors declare no competing interests.

\section{References}

1. Afanasyeva, M., D. Georgakopoulos, D.F. Belardi, A.C. Ramsundar, J.G. Barin, D.A. Kass, and N.R. Rose. 2004. Quantitative analysis of myocardial inflammation by flow cytometry in murine autoimmune myocarditis - correlation with cardiac function. Am. J. Pathol. 164:807-815.

2. Berti, R., A.J. Williams, J.R. Moffett, S.L. Hale, L.C. Velarde, P.J. Elliott, C.P. Yao, J.R. Dave, and F.C. Tortella. 2002. Quantitative real-time RT-PCR analysis of inflammatorygene expression associated with ischemia-reperfusion brain injury. J. Cereb. Blood Flow Metab. 22:1068-1079.

3. Osuchowski, M.F., J. Siddiqui, S. Copeland, and D.G. Remick. 2005. Sequential ELISA to profile multiple cytokines from small volumes. J. Immunol. Methods 302:172-181.

4. Lowe, K.C. 2003. Engineering blood: Synthetic substitutes from fluorinated compounds. Tissue Eng. 9:389-399.

5. Riess, J.G. 2005. Understanding the fundamentals of perfluorocarbons and perfluorocarbon emulsions relevant to in vivo oxygen delivery. Artif. Cells Blood Substit. Immobil. Biotechnol. 33:47-63.

6.Srinivas, M., M.S. Turner, J.M. Janjic, P.A. Morel, D.H. Laidlaw, and E.T. Ahrens. 2009. In vivo cytometry of antigen-specific T cells using F-19 MRI. Magn. Reson. Med. 62:747-753.

7. Helfer, B.M., A. Balducci, A.D. Nelson, J.M. Janjic, R.R. Gil, P. Kalinski, I.J.M. De Vries, E.T. Ahrens, and R.B. Mailliard. 2010. Functional assessment of human dendritic cells labeled for in vivo F-19 magnetic resonance imaging cell tracking. Cytotherapy 12:238-250.

8. Janjic, J.M. and E.T. Ahrens. 2009. Fluorinecontaining nanoemulsions for MRI cell tracking. Wiley Interdiscip. Rev. Nanomed. Nanobiotechnol. 1:492-501.

9. Srinivas, M., P.A. Morel, L.A. Ernst, D.H. Laidlaw, and E.T. Ahrens. 2007. Fluorine-19 MRI for visualization and quantification of cell migration in a diabetes model. Magn. Reson. Med. 58:725-734.

10. Ahrens, E.T., R. Flores, H.Y. Xu, and P.A. Morel. 2005. In vivo imaging platform for tracking immunotherapeutic cells. Nat. Biotechnol. 23:983-987.

11. Lorentzen, J.C., S. Issazadeh, M. Storch, M.I. Mustafa, H.Lassman, C. Linington, L. Klareskog, and T. Olsson. 1995. Protracted, relapsing and demyelinating experimental autoimmune encephalomyelitis in DA rats immunized with syngeneic spinal cord and incomplete Freund's adjuvant. J. Neuroimmunol. 63:193-205. 


\section{BioTechnioues}

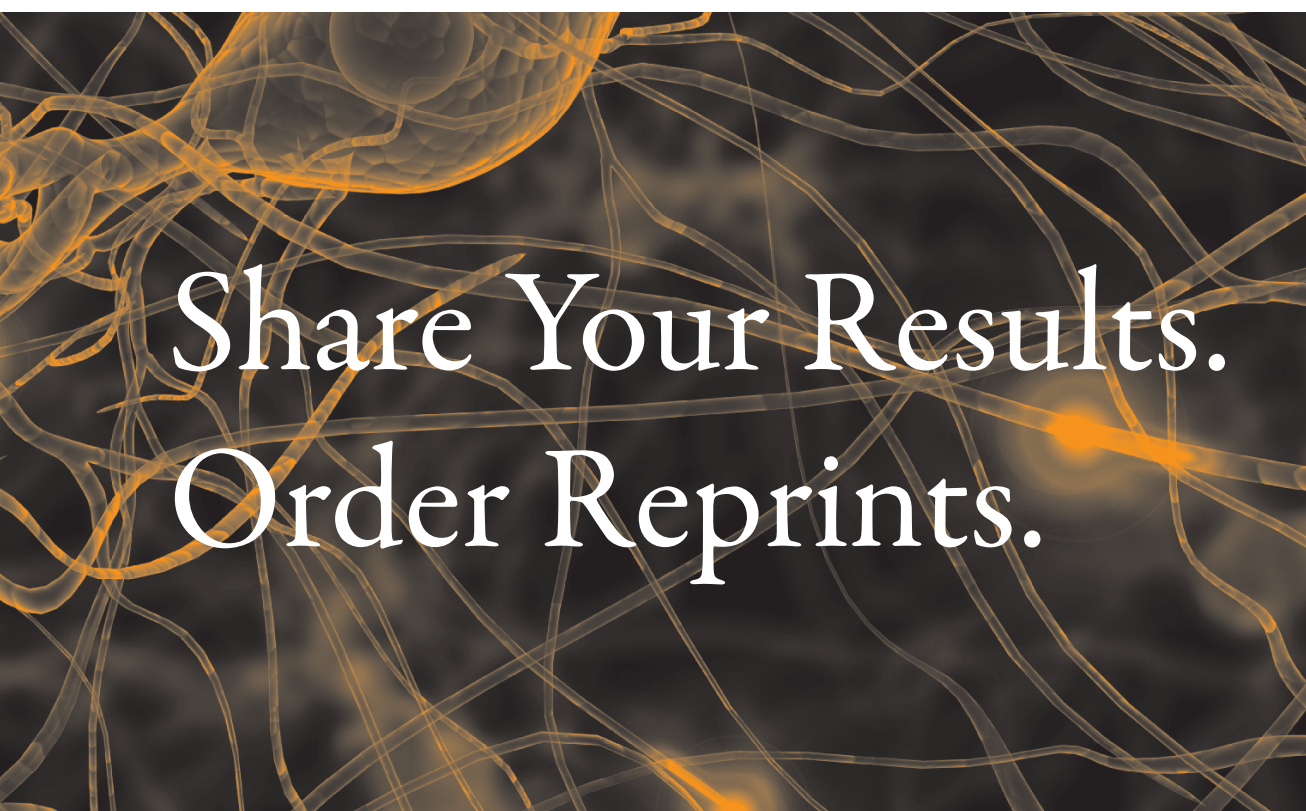

Author Reprints

Make the most of your hard work by ordering reprints of your article published in BioTechniques. Reprints are an inexpensive and easy way to distribute your findings to students and colleagues alike.

\section{Corporate Reprints}

Leverage BioTechniques, the most powerful brand in the market. Reprints help support your sales effort by utilizing articles that spotlight your brand/products to educate customers at meetings and industry trade events.

More info:

www.BioTechniques.com/reprints
12. Martin, R. and H.F. McFarland. 1995. Immunological aspects of experimental allergic encephalomyelitis and multiple sclerosis. Crit. Rev. Clin. Lab. Sci. 32:121-182.

13. Janjic, J.M., M. Srinivas, D.K.K. Kadayakkara, and E.T. Ahrens. 2008. Self-delivering nanoemulsions for dual fluorine-19 MRI and fluorescence detection. J. Am. Chem. Soc. 130:2832-2841.

14. Kraff, M.P. 2001. Fluorocarbons and fluorinated amphiphiles in drug delivery and biomedical research. Adv. Drug Deliv. Rev. 47:209-228.

15. Krafft, M.P. and J.G. Riess. 2007. Perfluorocarbons: Life sciences and biomedical uses: dedicated to the memory of Professor Guy Ourisson, a true RENAISSANCE man. J. Polym. Sci. Part A: Polym. Chem. 45:1185-1198.

16. Radu, C.G., C.J.Shu, S.M. Shelly, M.E. Phelps, and O.N. Witte. 2007. Positron emission tomography with computed tomography imaging of neuroinflammation in experimental autoimmune encephalomyelitis. Proc. Natl. Acad. Sci. USA 104:1937-1942.

17. Hitchens, T.K., Q. Ye, D.F. Eytan, J.M. Janjic, E.T.Ahrens, and C. Ho. 2011. 19F MRI detection of acute allograft rejection with in vivo perfluorocarbon labeling of immune cells. Magn. Reson. Med. [Epub ahead of print].

18. McGoron, A.J., R. Pratt, J.Zhang, Y. Shiferaw, S. Thomas, and R. Millard. 1994. Perfluorocarbon distribution to liver, lung and spleen of emulsions of perfluorotributylamine (FTBA) in pigs and rats and perfluorooctyl bromide (PFOB) in rats and dogs by F-19 NMR spectroscopy. Artif. Cells Blood Substit. Immobil. Biotechnol. 22:12431250.

19. Noth, U., S.P. Morrissey, R. Deichmann, S. Jung, H. Adolf, A. Haase, and J. Lutz. 1997. Perfluoro15-crown-5-ether labelled macrophages in adoptive transfer experimental allergic encephalomyelitis. Artif. Cells Substit. Immobil. Biotechnol. 25:243-254.

20. Lanza, G.M., P.M. Winter, A.M. Neubauer, S.D. Caruthers, F.D. Hockett, and S.A. Wickline. 2005. 1H/19F magnetic resonance molecular imaging with perfluorocarbon nanoparticles, pp. 58-78. In E.T. Ahrens (Ed.), In Vivo Cellular and Molecular Imaging. Elsevier, San Diego.

21. Flogel, U., Z. Ding, H. Hardung, S. Jander, G. Reichmann, C. Jacoby, R. Schubert, and J. Schrader. 2008. In vivo monitoring of inflammation after cardiac and cerebral ischemia by fluorine magnetic resonance imaging. Circulation 118:140-148.

22.Ebner, B., P. Behm, C. Jacoby, S. Burghoff, B.A. French, J. Schrader, and U. Flögel. 2010. Early assessment of pulmonary inflammation by $19 \mathrm{~F}$ MRI in vivo. Circ. Cardiovasc. Imaging 3:202-210.

23. Kadayakkara, D.K., P.L. Beatty, M.S. Turner, J.M. Janjic, E.T. Ahrens, and O.J. Finn. 2010. Inflammation driven by overexpression of the hypoglycosylated abnormal mucin 1 (MUC1) links inflammatory bowel disease and pancreatitis. Pancreas 39:510-515.

Received 8 June 2010; accepted 16 March 2011.

Address correspondence to Eric T. Ahrens, Department of Biological Sciences, Carnegie Mellon University, 4400 Fifth Avenue, Pittsburgh, PA, 15213, USA.e-mail: eta@cmu.edu

To purchase reprints of this article, contact: biotechniques@fosterprinting.com 